\title{
Extreme CO Isotopic Abundances in the ULIRG IRAS 13120-5453: An Extremely Young Starburst or Top-heavy Initial Mass Function
}

\author{
Kazimierz Sliwa ${ }^{1}$, Christine D. Wilson ${ }^{2}$, Susanne Aalto ${ }^{3}$, and George C. Privon ${ }^{4,5}$ \\ ${ }^{1}$ MPI for Astronomy, Königstuhl 17, D-69117 Heidelberg, Germany; sliwa@mpia-hd.mpg.de \\ 2 Department of Physics and Astronomy, McMaster University, Hamilton, ON L8S 4M1, Canada \\ ${ }^{3}$ Department of Earth and Space Sciences, Chalmers University of Technology, Onsala Space Observatory, SE-439 94 Onsala, Sweden \\ ${ }^{4}$ Instituto de Astrofśica, Facultad de Física, Pontificia Universidad Católica de Chile, Casilla 306, Santiago 22, Chile \\ ${ }^{5}$ Departamento de Astronomía, Universidad de Concepción, Casilla 160-C, Concepción, Chile \\ Received 2017 February 15; revised 2017 April 8; accepted 2017 April 21; published 2017 May 4
}

\begin{abstract}
We present ALMA ${ }^{12} \mathrm{CO}(J=1-0,3-2$ and $6-5),{ }^{13} \mathrm{CO}(J=1-0)$, and $\mathrm{C}^{18} \mathrm{O}(J=1-0)$ observations of the local ultraluminous infrared galaxy (ULIRG) IRAS 13120-5453. The morphologies of the three isotopic species differ, as ${ }^{13} \mathrm{CO}$ shows a hole in emission toward the center. We measure integrated brightness temperature line ratios of ${ }^{12} \mathrm{CO} /{ }^{13} \mathrm{CO} \geqslant 60$ (exceeding 200) and ${ }^{13} \mathrm{CO} / \mathrm{C}^{18} \mathrm{O} \leqslant 1$ in the central region. Assuming optical thin emission, $\mathrm{C}^{18} \mathrm{O}$ is more abundant than ${ }^{13} \mathrm{CO}$ in several regions. The abundances within the central 500 pc are consistent with the enrichment of the interstellar medium via a young starburst ( $<7 \mathrm{Myr}$ ), a top-heavy initial mass function, or a combination of both.
\end{abstract}

Key words: galaxies: abundances - galaxies: individual (IRAS 13120-5453) - galaxies: interactions - galaxies: starburst - radiative transfer - submillimeter: galaxies

\section{Introduction}

Isotopic abundances in the interstellar medium (ISM) can be used as a tracer of stellar nucleosynthesis. The ${ }^{12} \mathrm{C} /{ }^{13} \mathrm{C}$ isotope ratio is an important tracer of the relative degree of primary versus secondary processing in stars. The ${ }^{12} \mathrm{C}$ atom is a primary species produced in intermediate- and high-mass stars (e.g., Prantzos et al. 1996). Massive stars are also responsible for the majority of ${ }^{16} \mathrm{O}$ and ${ }^{18} \mathrm{O}$. The ${ }^{13} \mathrm{C}$ atom is an intermediary species that is transformed into ${ }^{14} \mathrm{~N}$. In the red giant phase of low/intermediate-mass stars, ${ }^{13} \mathrm{C}$ is lifted to the envelope via convection (Wilson \& Matteucci 1992) and eventually released into the ISM. Massive stars are short-lived and start to enrich the ISM in ${ }^{12} \mathrm{C}$ in $\sim 10^{6}$ years, while ${ }^{13} \mathrm{C}$ enrichment needs $\sim 10^{9}$ years (e.g., Vigroux et al. 1976).

Ultra/Luminous Infrared Galaxies (ULIRGs) are extreme starbursts offering great laboratories in which to study highmass star formation. It has long been observed that ${ }^{13} \mathrm{CO}$ emission is unusually weak relative to ${ }^{12} \mathrm{CO}(\sim 20-40$; Aalto et al. 1991; Casoli et al. 1992) compared to that from normal disk galaxies ( 10; e.g., Paglione et al. 2001). Pioneering work by Casoli et al. (1992) and Henkel \& Mauersberger (1993) presented several scenarios by which to explain this unusual emission ratio, such as: optical depth effects; abundance variations via some mechanism such as photo-dissociation, inflowing low-metallicity gas, or enrichment of the ISM; and a two-phase molecular medium consisting of a diffuse envelope where ${ }^{12} \mathrm{CO}$ can better self-shield than ${ }^{13} \mathrm{CO}$. Recent radiative transfer modeling of the molecular gas in several ULIRGs is consistent with high $\left[{ }^{12} \mathrm{CO}\right] /\left[{ }^{13} \mathrm{CO}\right]$ abundance ${ }^{6}$ ratios $(>90$; Sliwa et al. 2013, 2014; Henkel et al. 2014; Papadopoulos et al. 2014; Tunnard et al. 2015).

Recent work on the $\left[{ }^{16} \mathrm{O}\right] /\left[{ }^{18} \mathrm{O}\right]$ abundance in ULIRGs using Herschel $\mathrm{H}_{2} \mathrm{O}$ and $\mathrm{OH}$ observations have shown varying values from $\leqslant 30$ for Mrk231 (González-Alfonso et al. 2010), around

\footnotetext{
6 Square brackets denote an abundance ratio, while all other ratios are integrated brightness temperature ratios.
}

50-150 for Arp220 (González-Alfonso et al. 2014) and Zw 049.057 (Falstad et al. 2015), and $\geqslant 500$ for Arp 299 (Falstad et al. 2017) and NGC 4418 (González-Alfonso et al. 2014). König et al. (2016) used Atacama Large Millimeter/submillimeter Array (ALMA) $\mathrm{CO}$ data to show that the $\left[{ }^{16} \mathrm{O}\right] /\left[{ }^{18} \mathrm{O}\right] \geqslant 900$ for NGC 1614. The high $\left[{ }^{16} \mathrm{O}\right] /\left[{ }^{18} \mathrm{O}\right]$ abundance ratios are believed to be due to inflowing gas and the low values to stellar processing.

IRAS 13120-5453 (dubbed the "Yo-yo") is a nearby ULIRG $\left(D_{\mathrm{L}}=144 \mathrm{Mpc}\right)$ with a far-infrared luminosity $\left(L_{\mathrm{FIR}}=1.5 \times 10^{12} L_{\odot} ;\right.$ Sanders et al. 2003) similar to that of Arp 220. The system has been classified as a post-merger (Haan et al. 2011). X-ray emission is consistent with a Compton-thick active galactic nucleus (AGN; Iwasawa et al. 2011) and contributes $\sim 18 \%$ to the infrared luminosity (Sturm et al. 2011). The Herschel Fourier Transform Spectrometer (FTS) observed multiple high-J CO lines as well as C I, $\mathrm{H}_{2} \mathrm{O}, \mathrm{N}$ II, OH and more (Mashian et al. 2015; Rosenberg et al. 2015; Pearson et al. 2016; Privon et al. 2017). Privon et al. (2017) shows that the $\mathrm{HCN} / \mathrm{HCO}^{+}$line ratio observed with ALMA suggests an increased HCN abundance via turbulent heating.

In this Letter, we present new ALMA Cycle 2 observations of IRAS 13120-5453 where we have detected three ${ }^{12} \mathrm{CO}$ transitions, ${ }^{13} \mathrm{CO}$, and $\mathrm{C}^{18} \mathrm{O} J=1-0$. The morphology of the three species differs and offers insight into the mechanism that may be controlling the $\left[{ }^{12} \mathrm{CO}\right] /\left[{ }^{13} \mathrm{CO}\right]$ ratio. We show that massive stars have enriched the ISM in ${ }^{12} \mathrm{C}$ and ${ }^{18} \mathrm{O}$ and drive the observed line ratios in IRAS 13120-5453.

\section{Observations and Line Ratios}

ALMA was used to observe IRAS 13120-5453 in Cycle 2 using Bands 3, 7, and 9 (Table 1). We calibrated all data sets manually in CASA v4.5.3 (McMullin et al. 2007) using standard calibration steps. We implemented two iterations of phase-only self-calibration on the ${ }^{12} \mathrm{CO}$ data sets, which did not significantly alter the morphology of IRAS 13120-5453. We 
Table 1

Observational Data

\begin{tabular}{|c|c|c|c|c|c|}
\hline Parameter $^{\mathrm{a}}$ & ${ }^{12} \mathrm{CO} J=1-0$ & ${ }^{12} \mathrm{CO} J=3-2$ & ${ }^{12} \mathrm{CO} J=6-5$ & ${ }^{13} \mathrm{CO} J=1-0$ & $\mathrm{C}^{18} \mathrm{O} J=1-0$ \\
\hline Obs Data & 2015 Jul 03 & 2015 Jun 07 & 2015 Jun 09 & 2015 Jul 22 & 2015 Jul 22 \\
\hline \multirow[t]{4}{*}{ Calibrators } & J1107-4449 & J1427-4206 & J1256-0547 & J1107-4449 & J1107-4449 \\
\hline & $\mathrm{J} 1551-1755$ & Titan & J1427-421 & $\mathrm{J} 1427-421$ & J1427-421 \\
\hline & Titan & J1329-5608 & J1329-5608 & J1329-5608 & J1329-5608 \\
\hline & J1329-5608 & J1315-5334 & J1427-4206 & & \\
\hline Integration Time (s) & 652 & 867 & 1567 & 3117 & 3117 \\
\hline Median PWV (mm) & 2.4 & 0.63 & 0.37 & 3.2 & 3.2 \\
\hline Median $T_{\text {sys }}(\mathrm{K})$ & 64 & 135 & 984 & 82 & 82 \\
\hline Flux $^{\mathrm{b}}\left(\mathrm{Jy} \mathrm{km} \mathrm{s}^{-1}\right)$ & $126( \pm 2)[ \pm 13]$ & $1265( \pm 15)[ \pm 130]$ & $2460( \pm 25)[ \pm 370]$ & $2.21( \pm 0.05)[ \pm 0.2]$ & $2.1( \pm 0.05)[ \pm 0.2]$ \\
\hline rms $\left(\mathrm{mJy}\right.$ beam $\left.^{-1}\right)$ & $1.5\left(20 \mathrm{~km} \mathrm{~s}^{-1}\right)$ & $1.1\left(20 \mathrm{~km} \mathrm{~s}^{-1}\right)$ & $16\left(20 \mathrm{~km} \mathrm{~s}^{-1}\right)$ & $0.3\left(35 \mathrm{~km} \mathrm{~s}^{-1}\right)$ & $0.3\left(35 \mathrm{~km} \mathrm{~s}^{-1}\right)$ \\
\hline Resolution (arcsec) & $0.58 \times 0.35$ & $0.39 \times 0.29$ & $0.25 \times 0.16$ & $0.55 \times 0.41$ & $0.55 \times 0.41$ \\
\hline
\end{tabular}

Notes.

a Other lines present in the data will be discussed in a forthcoming paper (K. Sliwa et al., in preparation).

${ }^{\mathrm{b}}$ Uncertainties in curved and square brackets denote measurement and calibration uncertainties, respectively.

CLEAN the data cubes using a Briggs robust weighting of 0.5 down to $1 \sigma$ level with channels widths of 20 or $35 \mathrm{~km} \mathrm{~s}^{-1}$. Integrated intensity maps were created using the CPROPs (Rosolowsky \& Leroy 2006) masking routine ${ }^{7}$ and only channels that contained masked emission were included down to $1.5 \sigma$. All maps were primary-beam corrected (Figure 1).

All three ${ }^{12} \mathrm{CO}$ transitions have similar morphologies with a single nucleus.

The ${ }^{13} \mathrm{CO} J=1-0$ emission is more interesting, with no emission above $1.5 \sigma$ near the central nucleus $(\sim 0$ ". $85 \times 0$ "! $3=590 \mathrm{pc} \times 210 \mathrm{pc})$ and two relatively strong emission regions outside of the nucleus. The region lacking emission is within the starburst region $(0.5 \mathrm{kpc})$ measured by Privon et al. (2017). Interestingly, the $C^{18} \mathrm{O}$ emission is relatively strong where there is no ${ }^{13} \mathrm{CO}$ emission. In the central 0!" 3 spectrum (Figure 2), it is evident that $\mathrm{C}^{18} \mathrm{O}$ is stronger than ${ }^{13} \mathrm{CO}$. We note that $\mathrm{C}^{18} \mathrm{O}$ may be partially contaminated by HNCO $\left(5_{0,5}-4_{0.4}\right)$ and several higher-energy transitions that lie on top of the $\mathrm{C}^{18} \mathrm{O}$ line. Along a line of sight, the maximum contamination is likely $30 \%$ determined from the peak of the HNCO $\left(5_{0,5}-4_{0,4}\right)$ transition; however, we do not make any corrections since this is an upper limit to the contamination and may only contribute to part of the $\mathrm{C}^{18} \mathrm{O}$ line profile.

Selective UV photo-dissociation of the rare $\mathrm{CO}$ isotopologues should affect both ${ }^{13} \mathrm{CO}$ and $\mathrm{C}^{18} \mathrm{O}$, with the nominally rarer $\mathrm{C}^{18} \mathrm{O}$ affected the most. Thus, it could not produce the observed relative line intensity ratio variations between them, let alone boost the $\mathrm{C}^{18} \mathrm{O}$ abundance to be higher than ${ }^{13} \mathrm{CO}$ in the inner $500 \mathrm{pc}$ of IRAS 13120-5453. If optical depth effects were causing the ring, $\mathrm{C}^{18} \mathrm{O}$ should also be observed in a ring since both ${ }^{13} \mathrm{CO}$ and $\mathrm{C}^{18} \mathrm{O}$ are assumed to be optically thin. The Band 3 observations, observed 19 days apart, have similar $u v$ coverage, adding confidence to the observed differences in morphology and corresponding intensities between the lines. The three ${ }^{12} \mathrm{CO}$ maps (Figure 1) also show that as we go to a higher resolution $(J=1-0 \rightarrow J=6-5)$ we do not see a ring in ${ }^{12} \mathrm{CO}$.

Integrated brightness temperature $\left(I=\int T_{\mathrm{B}} d V\right)$ line ratio maps can offer some insight into the conditions of the

\footnotetext{
The routine finds pixels greater than $3 \sigma$ in two channels and then includes emission down to some $\sigma$ level around the pixel. This method is excellent at excluding spurious noise pixels.
}

molecular gas. We create the following line ratios maps:

$$
\begin{aligned}
R_{10} & =\frac{I^{12} \mathrm{CO}(1-0)}{I^{13} \mathrm{CO}(1-0)} \\
Y_{10} & =\frac{I^{13} \mathrm{CO}(1-0)}{I^{\mathrm{C}^{18} \mathrm{O}(1-0)}} \\
Z_{10} & =\frac{I^{12 \mathrm{CO}(1-0)}}{I^{\mathrm{C} 8 \mathrm{O}(1-0)}} .
\end{aligned}
$$

We match the ${ }^{12} \mathrm{CO},{ }^{13} \mathrm{CO}$, and $\mathrm{C}^{18} \mathrm{O} J=1-0$ maps to an angular resolution of 0 " $60 \times 0$." 45 . We then create line ratio maps by cutting emission below $2 \sigma$ in each map and converting the units from Jy beam ${ }^{-1} \mathrm{~km} \mathrm{~s}^{-1}$ to $\mathrm{K}\left(T_{B}\right) \mathrm{km} \mathrm{s}^{-1}$ (Figure 3).

The $R_{10}$ line ratio shows a wide range of values from $\sim 10$ to over 250 . While values of around 30 are common for local ULIRGs (e.g., Sliwa et al. 2012, 2013, 2014, 2017; Sliwa \& Downes 2017), values exceeding 100 have never been observed before in local ULIRGs. The $Y_{10}$ line ratio ranges from 0.2 to over 4 . Values below 1 are rare in extragalactic systems where normal disk galaxies have an average $Y_{10}$ value of $\sim 6$ and normal starbursts show $Y_{10}$ values of $\sim 3$ (JiménezDonaire et al. 2017). Arp 220 (Greve et al. 2009; Matsushita et al. 2009) and the high- $z$ ULIRG SMM J2135-0102 (Danielson et al. 2013) show a $Y_{10}$ ratio of 1 , while the LIRG merger remnant, NGC 2623, shows a $Y_{10}$ ratio of $\sim 1.8$ (K. Sliwa et al., in preparation). The $Z_{10}$ values range from $\sim 20-140$ and are similar to those of Mrk 231 (GonzálezAlfonso et al. 2010), Arp 220 (González-Alfonso et al. 2014), and $\mathrm{Zw} 049.057$ (Falstad et al. 2015).

\section{Extreme Isotopic Abundances}

We argue that IRAS 13120-5453 has an extreme isotopic abundance ratio when compared to normal star-forming galaxies. The brightness temperature line ratio of species A and $\mathrm{B}$ can be expressed as

$$
R=\frac{I^{\mathrm{A}}}{I^{\mathrm{B}}}=\frac{T_{\mathrm{ex}}^{\mathrm{A}}}{T_{\mathrm{ex}}^{\mathrm{B}}} \frac{\left(1-e^{-\tau_{\mathrm{A}}}\right)}{\left(1-e^{-\tau_{\mathrm{B}}}\right)}
$$

where $T_{\mathrm{ex}}$ is the excitation temperature and $\tau$ is the optical depth of the particular species and transition. For simplicity, we assume local thermal equilibrium (LTE; i.e., $T_{\mathrm{ex}}^{\mathrm{A}}=T_{\mathrm{ex}}^{\mathrm{B}}=T_{\text {kin }}$ ). (Significant radiative trapping for the abundant species can give $T_{\mathrm{ex}}^{\mathrm{A}}=T_{\text {kin }}$ while $T_{\mathrm{ex}}^{\mathrm{B}}<T_{\text {kin }}$.) If species $\mathrm{B}$ is optically thin and 


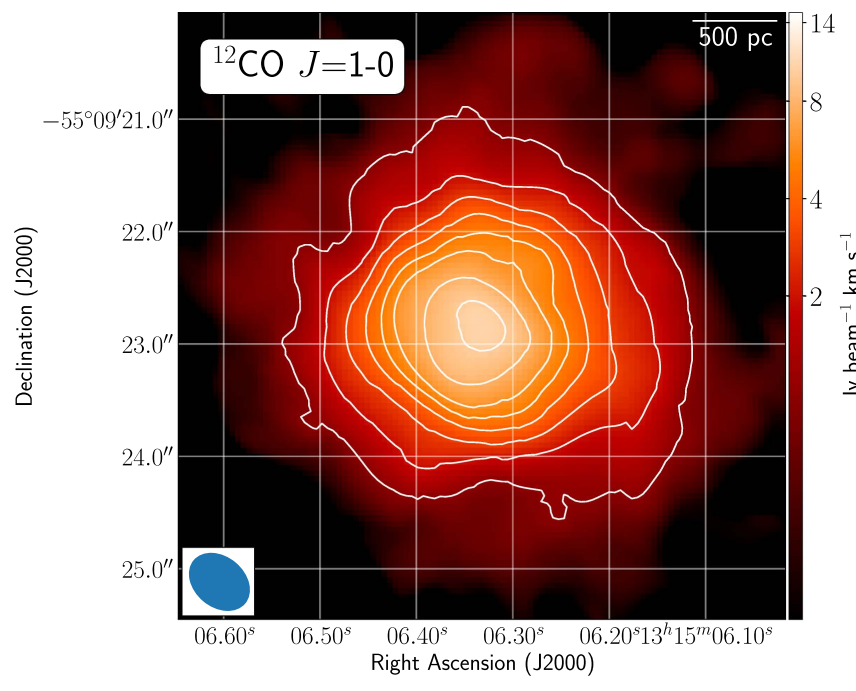

(a)

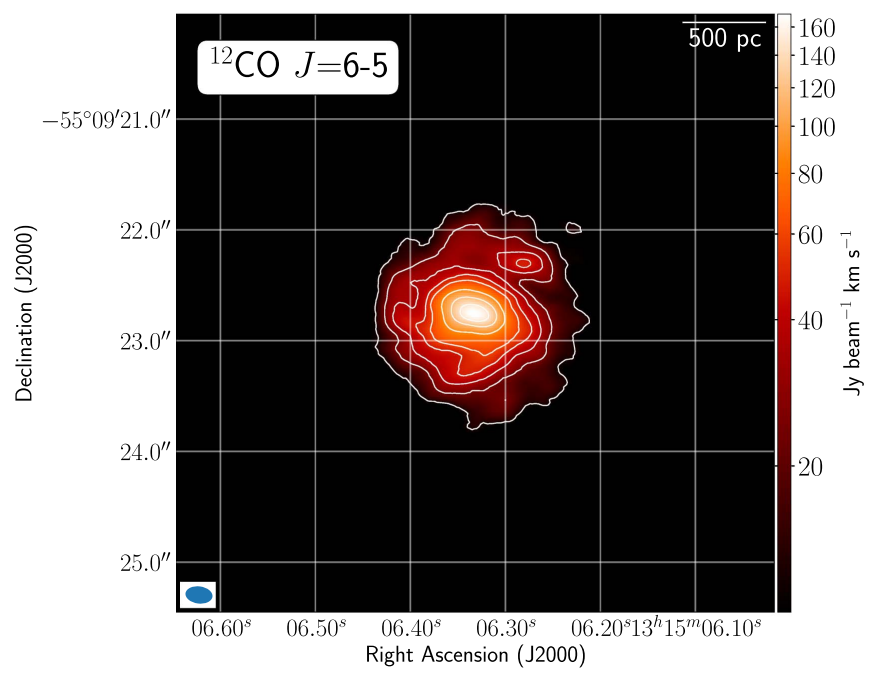

(c)

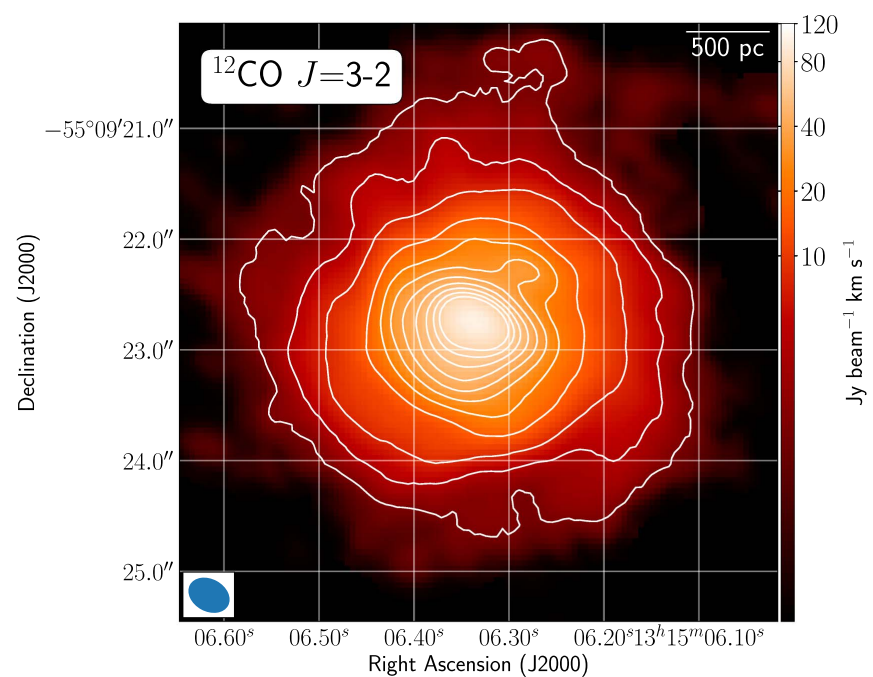

(b)

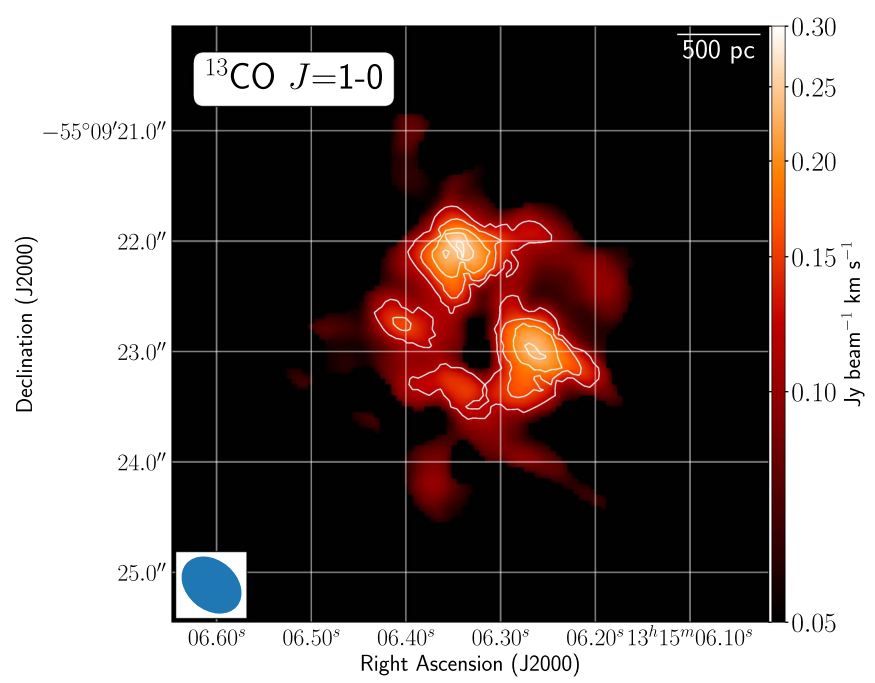

(d)

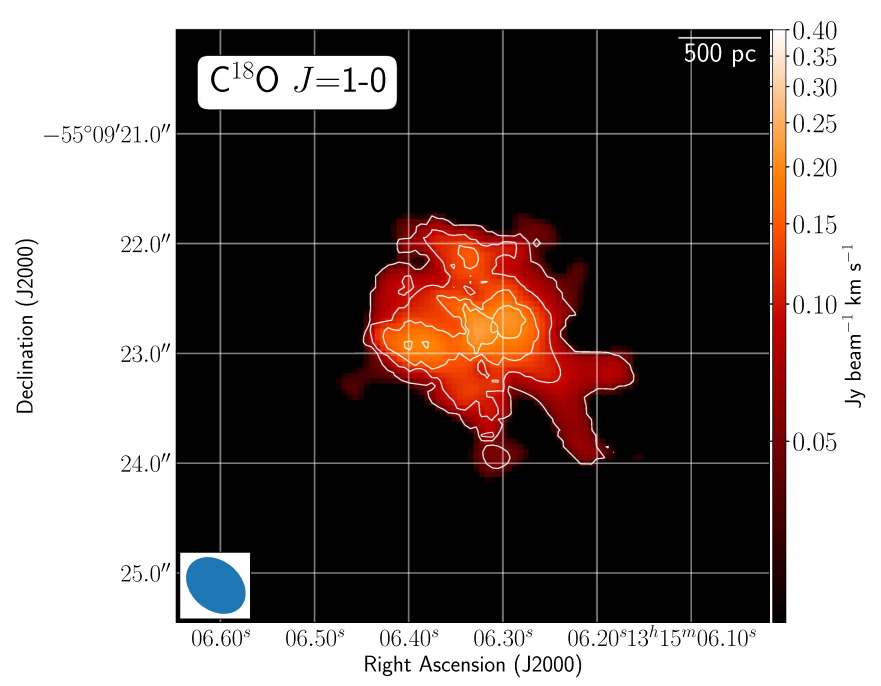

(e)

Figure 1. Integrated intensity maps for IRAS 13120-5453: (a) ${ }^{12} \mathrm{CO} J=1-0$, (b) ${ }^{12} \mathrm{CO} J=3-2$, (c) ${ }^{12} \mathrm{CO} J=6-5$, (d) ${ }^{13} \mathrm{CO} J=1-0$, and (e) $\mathrm{C}^{18} \mathrm{O} J=1-0$. The ellipse in the bottom-left corner represents the synthesized beam. 


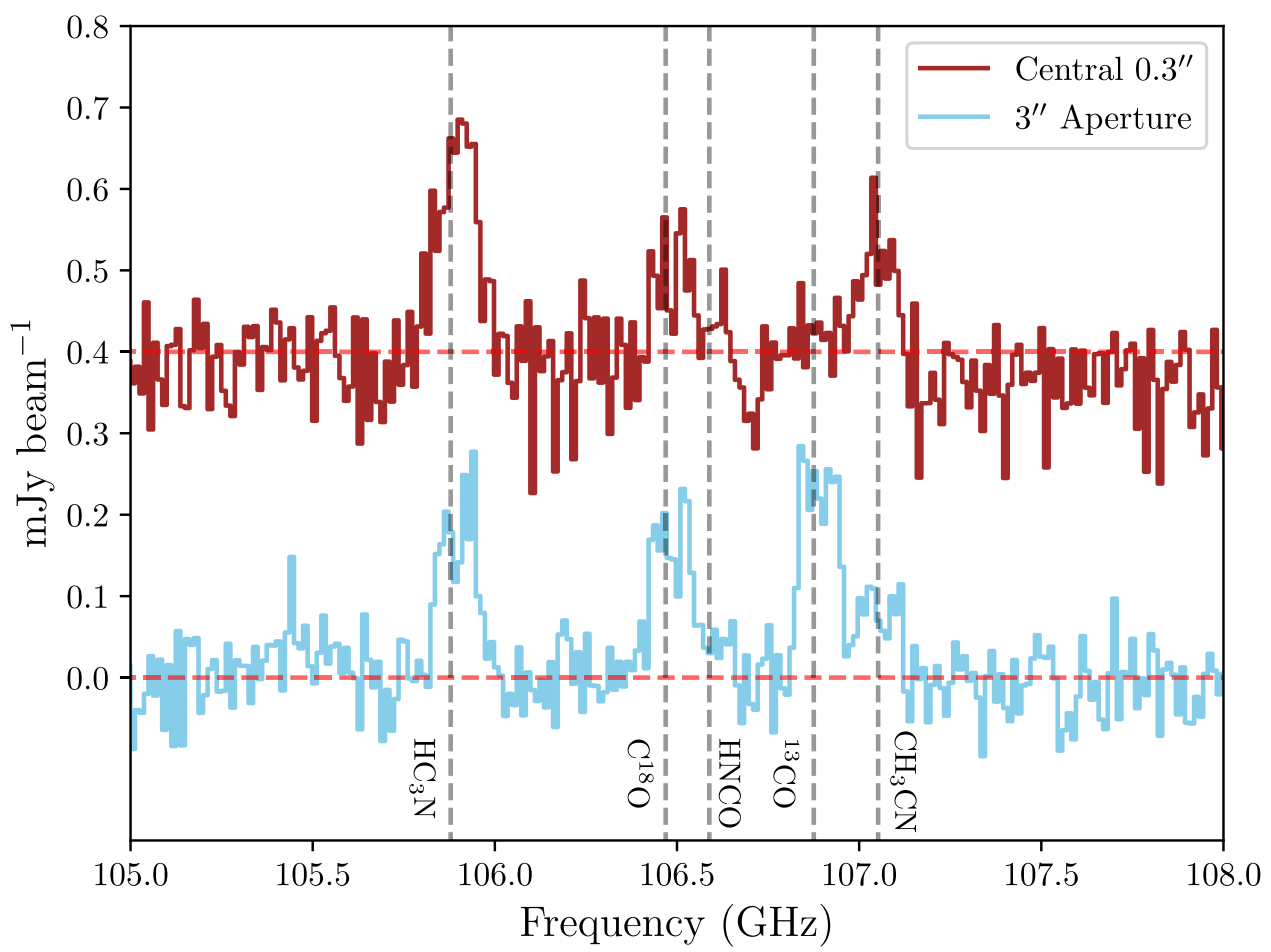

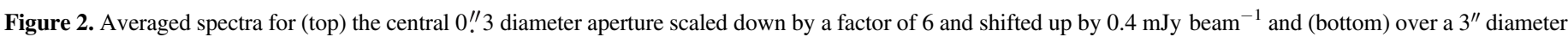
aperture. Identified spectral lines are marked with vertical dashed lines $(z=0.03112)$. For HNCO, we mark the $5_{0,5}-4_{0,4}$ transition.

species A is optically thick, then

$$
R \sim \frac{1}{\tau_{\mathrm{B}}} \sim \frac{[\mathrm{A}]}{[\mathrm{B}]} \frac{1}{\tau_{\mathrm{A}}}
$$

If ${ }^{12} \mathrm{CO}$ is optically thick, the line ratios $R_{10}$ and $Z_{10}$ are lower limits to the relative abundance of ${ }^{12} \mathrm{CO}$ to ${ }^{13} \mathrm{CO}$ and $\mathrm{C}^{18} \mathrm{O}$, since the observed line ratio is attenuated by the optical depth of ${ }^{12} \mathrm{CO}$. Since $R_{10}$ and $Z_{10} \gg 1$, both ${ }^{13} \mathrm{CO}$ and $\mathrm{C}^{18} \mathrm{O}$ must be optically thin. Thus, the $Y_{10}$ line ratio of $<1$ implies that $C^{18} \mathrm{O}$ is more abundant than ${ }^{13} \mathrm{CO}$ in the central region; this would still be true even if the $\mathrm{HNCO}$ contamination were to reach $50 \%$.

\section{Root of the Extreme Abundances}

Photo-dissociation: Since $\mathrm{C}^{18} \mathrm{O}$ is bright relative to ${ }^{13} \mathrm{CO}$, we can rule out selective photo-dissociation as the dominant mechanism, since both $\mathrm{C}^{18} \mathrm{O}$ and ${ }^{13} \mathrm{CO}$ would be destroyed by UV radiation. is

Fractionation: The most important carbon isotope exchange

$$
{ }^{13} \mathrm{C}^{+}+{ }^{12} \mathrm{CO} \rightleftharpoons{ }^{13} \mathrm{CO}+{ }^{12} \mathrm{C}^{+}+\Delta E \text {. }
$$

(Watson et al. 1976), where the forward reaction dominates in cold environments $(<30 \mathrm{~K})$ favoring the formation of ${ }^{13} \mathrm{CO}$. In hot environments, both directions have equal probability (Roueff et al. 2015). We use the non-LTE code RADEX (van der Tak et al. 2007) and a Bayesian likelihood code (Kamenetzky et al. 2012) to constrain the molecular gas physical conditions within the $\sim 400 \mathrm{pc}$ central region using only the ${ }^{12} \mathrm{CO}$ observations. We fit the ${ }^{12} \mathrm{CO} J=1-0$ line with a Gaussian profile of FWHM $=375 \mathrm{~km} \mathrm{~s}^{-1}$. The most probable solution is warm, dense molecular gas with a $T_{\text {kin }}=130_{-77}^{+400} \mathrm{~K}, \log$
$\left(n_{\mathrm{H}_{2}}\right)=4.2_{-0.0}^{+2.4} \mathrm{~cm}^{-3}$, and $\log \left(M\left(\mathrm{H}_{2}\right) / M_{\odot}\right)=7.8_{-0.1}^{+1.0}$. Since the molecular gas is not cold enough for the forward reaction to dominate, we can rule out fractionation as a possible mechanism affecting the abundance. We also note that $\mathrm{C}^{18} \mathrm{O}$ does not undergo fractionation and should reflect stellar processing (Langer et al. 1984).

Infalling Gas: The merger process can drive a gas inflow toward the nuclear regions (e.g., Hopkins et al. 2006; Kewley et al. 2006; Ellison et al. 2008). The Galaxy has an increasing radial gradient in the $\left[{ }^{12} \mathrm{CO}\right] /\left[{ }^{13} \mathrm{CO}\right]$ abundance ratio, ranging from 30 in the center to $>100$ at large radii (e.g., Milam et al. 2005). Jiménez-Donaire et al. (2017) have shown that a trend with $\left[{ }^{13} \mathrm{CO}\right] /\left[\mathrm{C}^{18} \mathrm{O}\right]$ exists in disk galaxies as well with an average value of $6.0 \pm 0.9$. Analyses of close galaxy pairs have shown that their metallicities are lower than similar field galaxies (Kewley et al. 2006; Ellison et al. 2008). Rupke et al. (2008) found that the dilution of the nuclear metallicity $(Z)$ due to gas inflow is $Z_{\text {final }} / Z_{\text {initial }} \sim 0.5$; therefore, if we assume an initial $\left[{ }^{12} \mathrm{CO}\right] /\left[{ }^{13} \mathrm{CO}\right]$ ratio of 30 , we would expect a final ratio of $\sim 60$. This would not be sufficient to explain the observed line ratios in the central regions, particularly the brighter $\mathrm{C}^{18} \mathrm{O}$ emission.

Nucleosynthesis: Enrichment of the ISM via massive stars is a likely mechanism. Massive stars are the dominant sources of ${ }^{12} \mathrm{C}$, ${ }^{16} \mathrm{O}$, and ${ }^{18} \mathrm{O}$, while ${ }^{13} \mathrm{C}$ is predominately released from low/intermediate-mass stars. Simulations show that the metallicity in the merger increases when the star formation rate increases significantly, especially near the end of the merger process (Torrey et al. 2012).

For nucleosynthesis enrichment to be plausible, the starburst must be young. With a normal initial mass function (IMF) such as the Kroupa IMF (e.g., Kroupa 2001) within $\sim 6 \mathrm{Myr}$, all stars $>30 M_{\odot}$ will have gone supernova, ejecting material. Using the nucleosynthesis yield calculations for core-collapse supernovae 


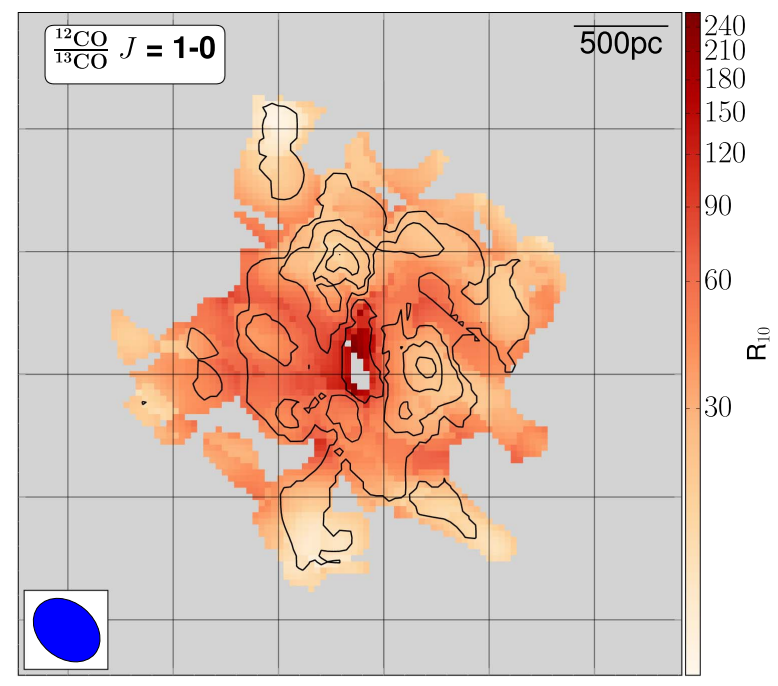

(a)

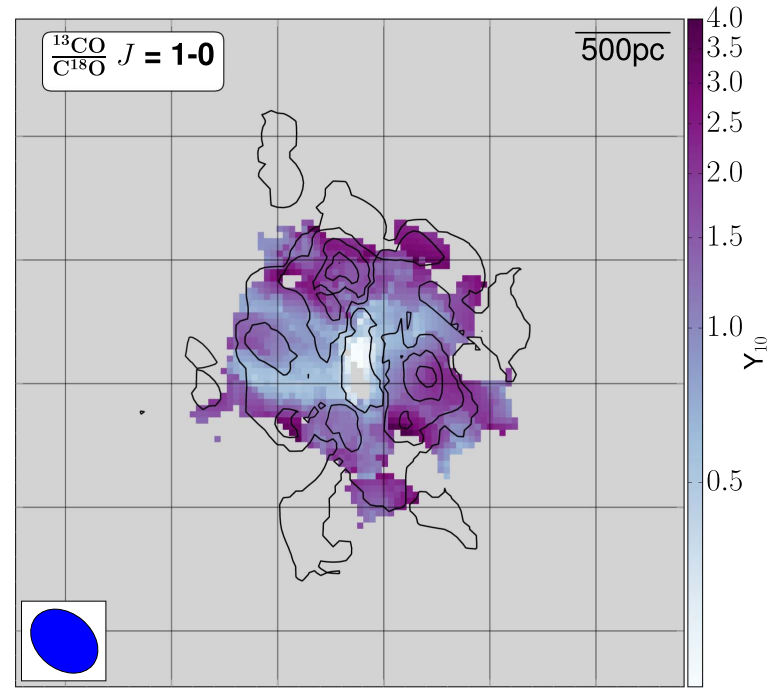

(b)

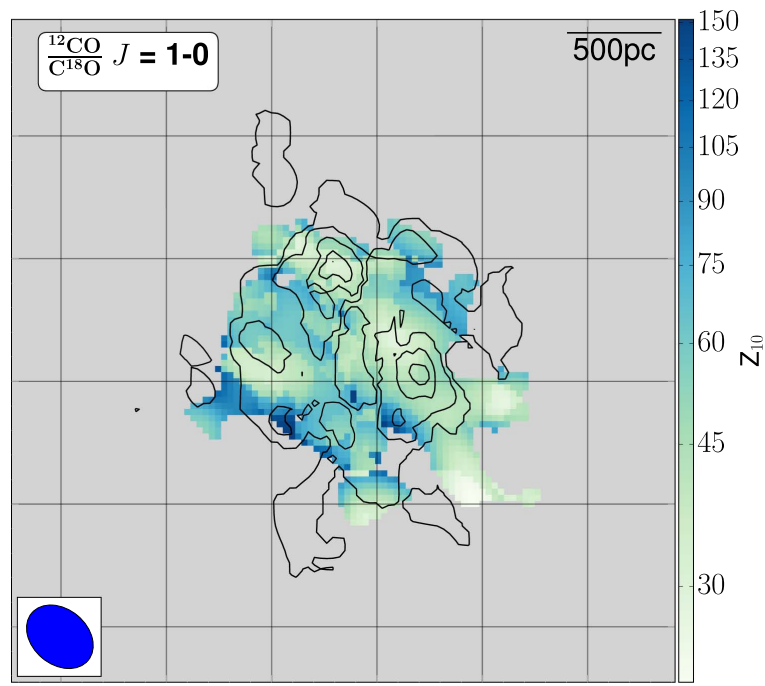

(c)

Figure 3. Line brightness temperature ratio maps for (a) $R_{10}$, (b) $Y_{10}$ and (c) $Z_{10}$. Each map has an angular resolution of $0 . " 6 \times 0$." 45 . Black contours are of ${ }^{13} \mathrm{CO} J=1-0$ added as a guide.
(CCSNe) of Nomoto et al. (2006) and assuming a total starforming molecular mass of $10^{9} M_{\odot}$ and an initial metallicity of $\mathrm{Z}=0.02\left(\sim \mathrm{Z}_{\odot}\right)$, the $\left[{ }^{12} \mathrm{CO}\right] /\left[{ }^{13} \mathrm{CO}\right]$ value of the ejected material after $6 \mathrm{Myr}$ will be $\sim 575$, while after $7 \mathrm{Myr}$, when all stars above $25 M_{\odot}$ will have gone supernova, the $\left[{ }^{12} \mathrm{CO}\right] /\left[{ }^{13} \mathrm{CO}\right]$ will be $\sim 60$.

If the starburst is older ( $>7 \mathrm{Myr}$ ), an alternative solution is a top-heavy IMF. Bartko et al. (2010) find a top-heavy IMF for the Galactic center of $d N / d m \propto m^{-0.45 \pm 0.3}$. Habergham et al. (2010) also invoke a top-heavy IMF to explain the excess of CCSNe in interacting/mergers galaxies when compared to isolated galaxies. Assuming that only stars of $10-130 M_{\odot}$ will eject material via $\mathrm{CCSNe}$, a Kroupa IMF will produce a $\left[{ }^{12} \mathrm{CO}\right] /\left[{ }^{13} \mathrm{CO}\right]$ abundance of 40 while a flat, top-heavy IMF $\left(\phi_{m} \propto m^{0}\right)$ will produce a $\left[{ }^{12} \mathrm{CO}\right] /\left[{ }^{13} \mathrm{CO}\right]$ abundance of 270 . While a flat IMF is an arbitrary choice, if the starburst is older than $\sim 6$ Myr a top-heavy IMF of some variety is required to explain our observed abundances in the central region. We also note that a combination of both a young-starburst and a topheavy IMF is also plausible. Future work into the star formation history of IRAS $13120-5453$ is required to clarify the starburst-age/IMF degeneracy.

We thank the referee for suggestions and comments that greatly improved this manuscript. K.S. thanks E. Schinnerer for useful discussions. This paper uses the following ALMA data: ADS/JAO.ALMA\#2012.1.00306.S + ADS/JAO.ALMA\#2013.1.00379.S. ALMA is a partnership of ESO (representing its member states), NSF (USA) and NINS (Japan), together with NRC (Canada) and NSC and ASIAA (Taiwan), and KASI (Republic of Korea), in cooperation with the Republic of Chile. The JAO is operated by ESO, AUI/NRAO, and NAOJ. The NRAO is a facility of the NSF operated under cooperative agreement by Associated Universities, Inc. G.C.P. was supported by a FONDECYT Postdoctoral Fellowship (No. 3150361).

Facility: ALMA.

\section{References}

Aalto, S., Johansson, L. E. B., Booth, R. S., \& Black, J. H. 1991, A\&A, 249, 323

Bartko, H., Martins, F., Trippe, S., et al. 2010, ApJ, 708, 834

Casoli, F., Dupraz, C., \& Combes, F. 1992, A\&A, 264, 55

Danielson, A. L. R., Swinbank, A. M., Smail, I., et al. 2013, MNRAS, 436, 2793

Ellison, S. L., Patton, D. R., Simard, L., \& McConnachie, A. W. 2008, AJ, 135,1877

Falstad, N., González-Alfonso, E., Aalto, S., et al. 2015, A\&A, 580, A52

Falstad, N., González-Alfonso, E., Aalto, S., \& Fischer, J. 2017, A\&A, 597, A105

González-Alfonso, E., Fischer, J., Graciá-Carpio, J., et al. 2014, A\&A, 561, A27

González-Alfonso, E., Fischer, J., Isaak, K., et al. 2010, A\&A, 518, L43

Greve, T. R., Papadopoulos, P. P., Gao, Y., \& Radford, S. J. E. 2009, ApJ, 692, 1432

Haan, S., Surace, J. A., Armus, L., et al. 2011, AJ, 141, 100

Habergham, S. M., Anderson, J. P., \& James, P. A. 2010, ApJ, 717, 342

Henkel, C., Asiri, H., Ao, Y., et al. 2014, A\&A, 565, A3

Henkel, C., \& Mauersberger, R. 1993, A\&A, 274, 730

Hopkins, P. F., Hernquist, L., Cox, T. J., et al. 2006, ApJS, 163, 1

Iwasawa, K., Sanders, D. B., Teng, S. H., et al. 2011, A\&A, 529, A106

Jiménez-Donaire, M. J., Cormier, D., Bigiel, F., et al. 2017, ApJL, 836, L29

Kamenetzky, J., Glenn, J., Rangwala, N., et al. 2012, ApJ, 753, 70

Kewley, L. J., Geller, M. J., \& Barton, E. J. 2006, AJ, 131, 2004

König, S., Aalto, S., Muller, S., et al. 2016, A\&A, 594, A70

Kroupa, P. 2001, MNRAS, 322, 231 
Langer, W. D., Graedel, T. E., Frerking, M. A., \& Armentrout, P. B. 1984, ApJ, 277, 581

Mashian, N., Sturm, E., Sternberg, A., et al. 2015, ApJ, 802, 81

Matsushita, S., Iono, D., Petitpas, G. R., et al. 2009, ApJ, 693, 56

McMullin, J. P., Waters, B., Schiebel, D., Young, W., \& Golap, K. 2007, in ASP Conf. Ser. 376, Astronomical Data Analysis Software and Systems XVI, ed. R. A. Shaw, F. Hill, \& D. J. Bell (San Francisco, CA: ASP), 127 Milam, S. N., Savage, C., Brewster, M. A., Ziurys, L. M., \& Wyckoff, S. 2005, ApJ, 634, 1126

Nomoto, K., Tominaga, N., Umeda, H., Kobayashi, C., \& Maeda, K. 2006, NuPhA, 777, 424

Paglione, T. A. D., Wall, W. F., Young, J. S., et al. 2001, ApJS, 135, 183

Papadopoulos, P. P., Zhang, Z.-Y., Xilouris, E. M., et al. 2014, ApJ, 788, 153

Pearson, C., Rigopoulou, D., Hurley, P., et al. 2016, ApJS, 227, 9

Prantzos, N., Aubert, O., \& Audouze, J. 1996, A\&A, 309, 760

Privon, G. C., Aalto, S., Falstad, N., et al. 2017, ApJ, 835, 213

Rosenberg, M. J. F., van der Werf, P. P., Aalto, S., et al. 2015, ApJ, 801, 72

Rosolowsky, E., \& Leroy, A. 2006, PASP, 118, 590
Roueff, E., Loison, J. C., \& Hickson, K. M. 2015, A\&A, 576, A99

Rupke, D. S. N., Veilleux, S., \& Baker, A. J. 2008, ApJ, 674, 172

Sanders, D. B., Mazzarella, J. M., Kim, D.-C., Surace, J. A., \& Soifer, B. T. 2003, AJ, 126, 1607

Sliwa, K., \& Downes, D. 2017, A\&A, in press (arXiv:1704.03766)

Sliwa, K., Wilson, C. D., Iono, D., Peck, A., \& Matsushita, S. 2014, ApJL, 796, L15

Sliwa, K., Wilson, C. D., Krips, M., et al. 2013, ApJ, 777, 126

Sliwa, K., Wilson, C. D., Matsushita, S., et al. 2017, ApJ, 840, 8

Sliwa, K., Wilson, C. D., Petitpas, G. R., et al. 2012, ApJ, 753, 46

Sturm, E., González-Alfonso, E., Veilleux, S., et al. 2011, ApJL, 733, L16

Torrey, P., Cox, T. J., Kewley, L., \& Hernquist, L. 2012, ApJ, 746, 108

Tunnard, R., Greve, T. R., Garcia-Burillo, S., et al. 2015, ApJ, 815, 114

van der Tak, F. F. S., Black, J. H., Schöier, F. L., Jansen, D. J., \& van Dishoeck, E. F. 2007, A\&A, 468, 627

Vigroux, L., Audouze, J., \& Lequeux, J. 1976, A\&A, 52, 1

Watson, W. D., Anicich, V. G., \& Huntress, W. T., Jr. 1976, ApJL, 205, L165

Wilson, T. L., \& Matteucci, F. 1992, A\&ARv, 4, 1 